\title{
Authors' reply re: Vascular Control by Infrarenal Aortic Cross-Clamping in Placenta Accreta Spectrum Disorders: description of technique. (Response to BJOG-20-2523)
}

\author{
Min-Min Chou ${ }^{1}$, Hsiu-Wei Su${ }^{2}$, Ming-Jer Chen², Hsiao-Fan Kung ${ }^{2}$, Jenn-Jhy Tseng ${ }^{2}$, \\ Wei-Chih Chen ${ }^{2}$, and Ya-Fang Chen ${ }^{2}$ \\ ${ }^{1}$ China Medical University Hospital \\ ${ }^{2}$ Taichung Veterans General Hospital
}

January 27, 2021

Letter to the Editor, BJOG Exchange

Authors' reply re: Vascular Control by Infrarenal Aortic Cross-Clamping in Placenta Accreta Spectrum Disorders: description of technique

Sir,

We thank Nieto-Calvache et al. for their insightful comments (1) on our article (2). We agree with their point that the choice of vascular control techniques should be determined by the topography and severity of PAS disorders. We only performed aortic cross-clamping to selected patients who were expected to bleed heavily during the cesarean hysterectomy by prenatal ultrasonography. However, it is always possible that prenatal imaging findings do not fully predict surgical conditions. Although planning ahead, the final decision to perform aortic cross-clamping (ACC) should be made during the cesarean hysterectomy, after visualizing an extraordinary volume of tortuous vessels at the lower uterine segment, parametrium, and/or bladder invasion with uterovesical hypervascularity. If the PAS disorder is falsely diagnosed, or the severity is less than predicted, the obstetricians can simply proceed with conventional cesarean delivery or hysterectomy without applying any additional procedure. The routine use of ACC techniques in patients with mild PAS disorders is not recommended.

Balloon occlusion of the infrarenal abdominal aorta theoretically provides the same level of pelvic perfusion blockage as our approach (3). A major disadvantage of this procedure is that the catheter must be placed before commencing the surgery (3), which may not be possible if a prenatal diagnosis of PAS disorder is not made. On the other hand, if the severity of PAS is overestimated, both the mother and the fetus receive unnecessary radiation exposure from fluoroscopic catheter insertion.

We do not have statistical data regarding additional PAS cases treated without aortic cross-clamping in the same period. We conducted a comparison between the subjects in this case series and our previouslypublished historical controls (4). A potentially significant decrease in surgical blood loss was found (2295.6 $\pm 2126.1 \mathrm{~mL}$ with ACC vs. $4445.7 \pm 3728.5 \mathrm{~mL}$ without intervention, $\mathrm{p}=0.0084)$. Nevertheless, we acknowledge this vague comparison without RCT is not scientifically robust enough to draw a definite conclusion. PAS disorders encompass a heterogeneous group of conditions characterized by different severity, extent of invasion, and associated hypervascularity. All these factors influence surgical difficulty and blood loss. Comparison of operative blood loss in different series will subject to bias.

In our case series, six patients bled more than 4 liters because they were the most severe cases with extensive 
uterovesical hypervascularity. Even with ACC, the surgeries were still challenging, especially in dissecting the bladder wall away from the extremely hypervascular lower uterine segment. The surgical procedures were prolonged and extensively neovascularization couldn't be completely occluded due to the three anastomotic pathways outside the vascular control provided by ACC (2).

An experienced multidisciplinary team approach (3) and delayed hysterectomy at 4 to 6 weeks interval after cesarean delivery may represent two of the optimum strategies for minimizing the degree of hemorrhage in the most severe patients; the latter approach allows time for uterine blood flow to decrease and for the placenta to regress from surrounding structures in situations in which immediate hysterectomy is deemed too dangerous (5).

M-M Chou ${ }^{\mathrm{a}, \mathrm{b}}, \mathrm{H}-\mathrm{W} \mathrm{Su}^{\mathrm{b}}$, M-J Chen ${ }^{\mathrm{b}}$, H-F Kung ${ }^{\mathrm{b}}$, J-J Tseng ${ }^{\mathrm{b}}$, W-C Chen ${ }^{\mathrm{b}}$, Y-F Chen ${ }^{\mathrm{b}}$

${ }^{a}$ Center for High-Risk Pregnancy and Maternal-Fetal Medicine, Departments of Obstetrics and Gynecology, China Medical University Hospital, Taichung, Taiwan

b Department of Obstetrics, Gynecology \& Women's Health, Taichung Veterans General Hospital, Taichung, Taiwan

\section{References}

1. Nieto-Calvache A, Rodriguez H, Ordoñez C. Re: Vascular Control by Infrarenal Aortic Cross-Clamping in Placenta Accreta Spectrum Disorders: description of technique. (First comment letter. Reference to be added).

2. Chou MM, Chen MJ, Su HW, Chan CW, Kung HF, Tseng JJ, et al. Vascular Control by Infrarenal Aortic Cross-Clamping in Placenta Accreta Spectrum Disorders: description of technique. BJOG. 2020 Nov 29; https://doi.org/10.1111/1471-0528.16605.

3. Kingdom JC, Hobson SR, Murji A, Allen L, Windrim RC, Lockhart E, et al. Minimizing surgical blood loss at cesarean hysterectomy for placenta previa with evidence of placenta increta or placenta percreta: the state of play in 2020. Am J Obstet Gynecol. 2020 Jan 30;223(3):322-329.

4. Chou MM, Ho ES, Lee YH. Prenatal diagnosis of placenta previa accreta by transabdominal color Doppler ultrasound. Ultrasound Obstet Gynecol. 2000 Jan;15(1):28-35.

5. Zuckerwise LC, Craig AM, Newton JM, Zhao S, Bennett KA, Crispens MA. Outcomes following a clinical algorithm allowing for delayed hysterectomy in the management of severe placenta accreta spectrum. Am J Obstet Gynecol. 2020;222(2):179.e1-179.e9. 